\title{
Large and Giant Middle to Lower Basilar Trunk Aneurysms Treated by Surgical and Interventional Neuroradiological Methods
}

\author{
Waro TAKI, Ichiro NAKAHARA*, Nobuyuki SAKAI, Keiko IRIE**, \\ Kenichi MURAO***, Naoki OHKATA ${ }^{\dagger}$, Masato TANAKA ${ }^{\dagger \dagger}$, \\ and Haruhiko KIKUCHI* \\ Department of Neurosurgery, Kyoto University Medical School, Kyoto; \\ *Department of Neurosurgery, National Cardiovascular Center, Suita, Osaka; \\ ${ }^{* *}$ Department of Neurosurgery, Kagawa Medical School, Kagawa; \\ ***Department of Neurosurgery, Mie University Medical School, Tsu; \\ ${ }^{\dagger}$ Department of Neurosurgery, Kin-I-Kyo Central Hospital, Sapporo; \\ ${ }^{\dagger}$ Department of Neurosurgery, Toho University Sakura Hospital, Sakura, Chiba
}

\begin{abstract}
Treatment of large and giant aneurysms of the basilar artery remains difficult and controversial. Three large or giant aneurysms of the lower basilar artery were treated with a combination of surgical and interventional neuroradiological procedures. All patients underwent the balloon occlusion test with hypotensive challenge (blood pressure reduced to $70 \%$ of the control value). The third patient did not tolerate the test. In the first patient, both vertebral arteries were occluded through a craniotomy. In the second patient, both the aneurysm and the basilar artery were occluded by detached balloons. In the third patient, one vertebral artery was occluded by surgical clipping and the other by detached helical coils and fiber coils. In spite of anti-coagulation and anti-platelet therapy, postoperative thrombotic or embolic ischemia occurred in the second and third patients. Fibrinolytic therapy promptly corrected the ischemic symptoms, but the second patient developed hemorrhagic complications at the craniotomy area 2 hours later. At follow-up examination, the first patient had only 8 th cranial nerve paresis, the second patient who had a hemorrhagic complication was bed-ridden, and the third patient had no deficit. Interventional occlusion requires a longer segment of the parent artery compared to surgical occlusion of the parent artery and might cause occlusion of the perforating arteries. However, selected use of various coils can occlude only a short segment of the parent artery. Thus, the postoperative management of thromboembolic ischemia after the occlusion of the parent artery is easier using the interventional technique.
\end{abstract}

Key words: cerebral aneurysm, interventional neuroradiology, craniotomy, basilar artery, giant aneurysm

\section{Introduction}

Large or giant aneurysms located at the middle to lower basilar artery are surrounded by the clivus, brain stem, and cranial nerves, and are very difficult to clip through the very narrow operative field even when extensive craniotomy is performed. ${ }^{3,14,17)}$ The subtemporal transtentorial, presigmoidal suboccipital, far lateral, and transoral approaches have all been used, but the operative field is not adequate when the aneurysm is large ${ }^{7,8,16)}$ Extracorporeal cir-

Received March 17, 1998; Accepted July 30, 1998 culatory assist and circulatory standstill under profound hypothermia might be of use for as long as 1 hour, ${ }^{2,4,11,12)}$ but a series of procedures such as aneurysmal puncture, endarterectomy of the aneurysm, and neck clipping must be carried out in this time. Therefore, successful neck clipping of large and giant aneurysms of the middle to lower basilar artery is still unusual. Parent artery occlusion before surgery or intravascular treatment must be considered as an alternative treatment. ${ }^{19.10)}$ In addition, surgical intervention in this anatomical location results in either transient or permanent cranial nerve paresis in the majority of patients. The coil 
occlusion technique of the aneurysmal cavity was recently introduced. This minimally invasive treatment can be repeated and does not cause impairment of the surrounding cranial nerves. This technique is generally successful when the aneurysm neck is smaller than $4 \mathrm{~mm}$ while broad-necked aneurysms are still difficult to treat. ${ }^{5,6)}$ Permanent parent arterial occlusion might result in thrombotic occlusion of the perforating branches and/or larger branches such as the anterior inferior cerebellar artery. Thus postoperative management to prevent such embolic occlusion or thrombotic occlusion is important.

We describe three cases of large and giant aneurysm treated by a combination of surgical and interventional neuroradiological procedures.

\section{Materials and Methods}

Four of 24 patients presenting with large or giant aneurysm of the basilar artery in the last 9 years at our hospital had the aneurysm located at the basilar trunk or the vertebral junction. Three patients were treated surgically. A 55-year-old male was referred to our hospital because of progressive gait disturbance, swallowing disturbance, and double vision. The patient and his family did not desire any intervention. The patient was followed up at another hospital, but he died because of aneurysmal rupture 5 months later.

Case 1: A 48-year-old female experienced transient loss of consciousness which lasted for 5 minutes. After she regained consciousness, she complained of transient nausea and vomited. Computed tomography (CT) at an emergency hospital showed no subarachnoid hemorrhage, but a large aneurysm was located anterior to the brain stem. Angiography revealed a large aneurysm at the origin of the basilar artery (Fig. 1). She had had subarachnoid hemorrhage 10 years previously due to ruptured left middle cerebral artery saccular aneurysms, which had been surgically clipped at another hospital. Angiography at that time disclosed fenestration of the vertebral artery at its junction but no apparent aneurysm. She was referred to our hospital 2 months later.

On admission, she was free of neurological symptoms. Angiography showed a large aneurysm of 20 $\mathrm{mm}$ maximum diameter at the vertebral junction where the fenestration had been found previously (Fig. 2). The aneurysm was directed laterally to the left. CT and magnetic resonance (MR) imaging showed that the aneurysm had compressed the pons and medulla oblongata. The aneurysmal neck was very wide and neck clipping was considered difficult. Prior to surgery, the occlusion test was performed with balloon catheters in the bilateral vertebral arteries just proximal to the bilateral posterior inferior cerebellar arteries (Fig. 3). The occlusion test was performed for 15 minutes when the blood pressure was normal and also for 15 minutes during hypotension induced by administration of ganglion blocker. The systolic pressure was lowered to 80-90 mmHg. The hexamethyl-propyleneamine oxime (HMPAO) regional cerebral blood flow ( $\mathrm{rCBF}$ ) image was obtained during hypotensive challenge. The patient tolerated these occlusion tests very well, and the change in rCBF was minimal. A microcatheter was directed up to the aneurysmal lumen through the right vertebral artery for possible occlusion of the aneurysmal cavity. The distal portion of a Guglielmi detachable coil (Target Inc., Fremont, Calif., U.S.A.) was inserted into the cavity, but the

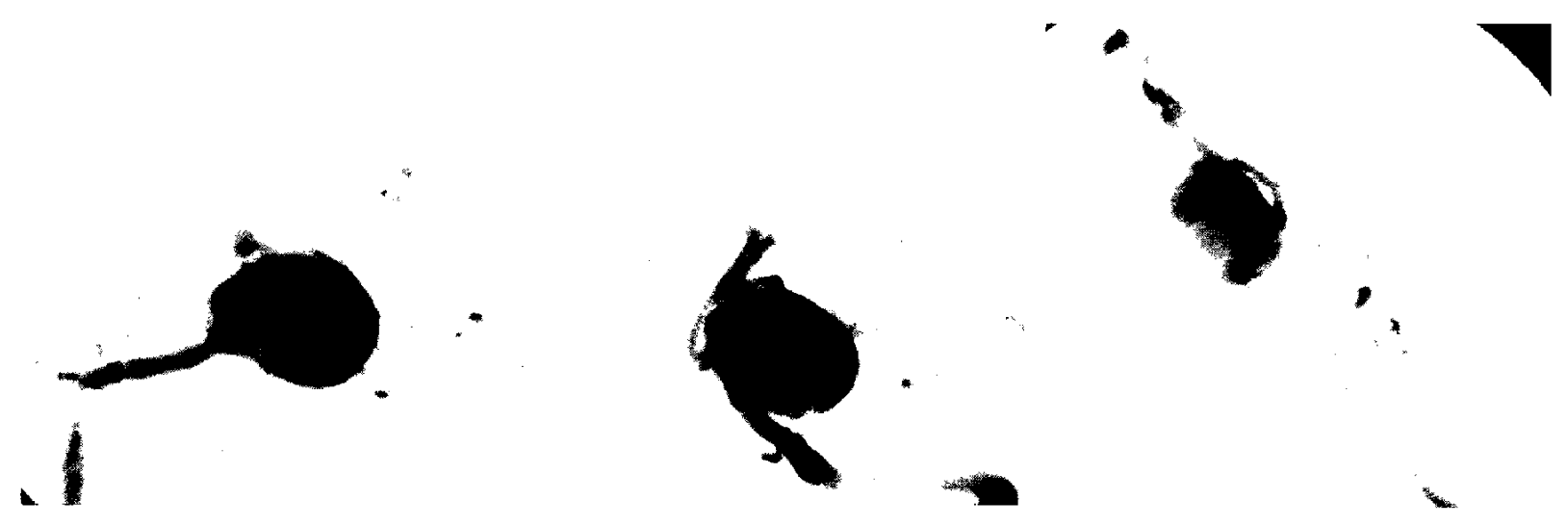

Fig. 1 Case 1. Preoperative angiograms revealing a large aneurysm at the fenestration of the vertebral junction. left: Left vertebral angiogram, anteroposterior view; center: right vertebral angiogram, anteroposterior view; right: left vertebral angiogram, lateral view. 


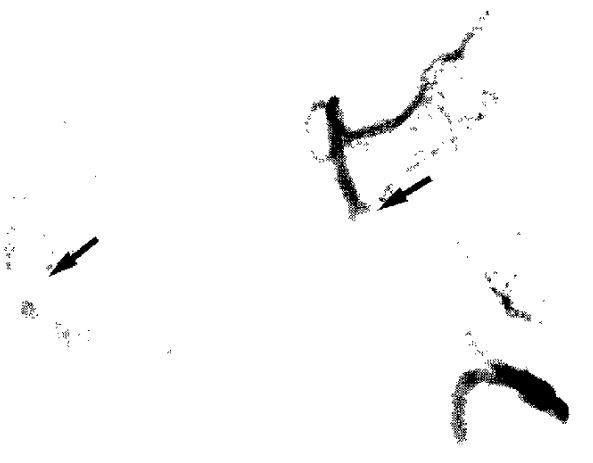

Fig. 2 Case 1. Left vertebral angiograms 10 years prior to the present admission showing a tiny bulge (arrow) at the fenestration of the basilar artery. left: Anteroposterior view, right: lateral view.

coil protruded into the parent artery and was withdrawn.

Surgical clipping of the aneurysm with temporary occlusion was possible since the patient could tolerate vertebral arterial occlusion. A right suboccipital craniotomy was performed. The cerebellum was retracted and the lateral medullary cistern opened to expose the right vertebral artery and the ventral surface of the aneurysm distal to the cranial nerves. The aneurysm was gradually dissected from the surrounding structure, but the aneurysm ruptured during the dissection of the ventral part, so a long straight clip was applied to the dome for hemostasis. Neck clipping was attempted but the parent basilar artery was difficult to spare. Because a part of the

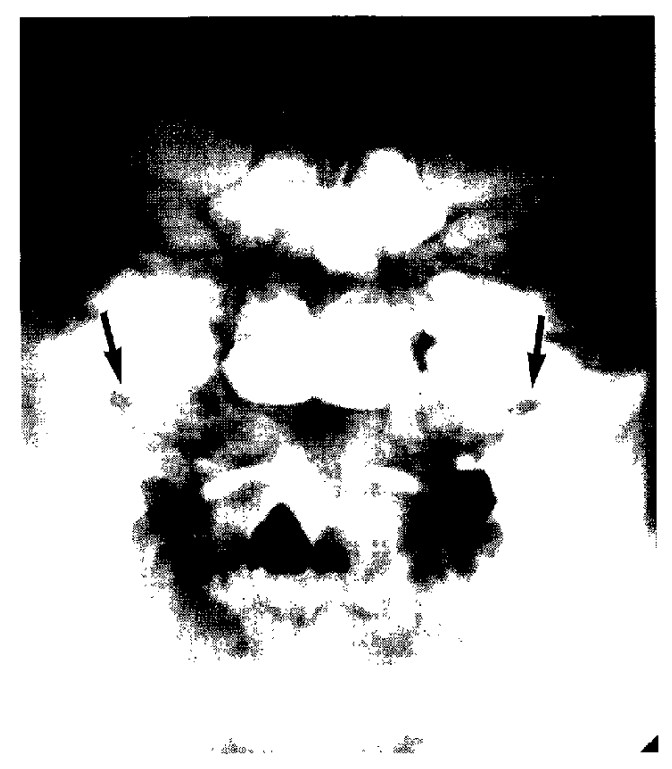

Fig. 3 Case 1. Skull radiograph, anteroposterior view, showing the two balloons temporarily occluding the bilateral (arrows) vertebral arteries in the occlusion test.

dome still remained unclipped, the bilateral parent vertebral arteries were clipped just distal to the bilateral posterior inferior cerebellar arteries.

Postoperatively, the patient had right abducens nerve paresis and right 8 th, 9th, 10th, and 11 th cranial nerve pareses. Anticoagulation therapy was not used for fear of postoperative hemorrhage at the surgical site. Low molecular weight dextran was administered as a potential hemodynamic assistant for microcirculatory improvement. The swallowing dis-
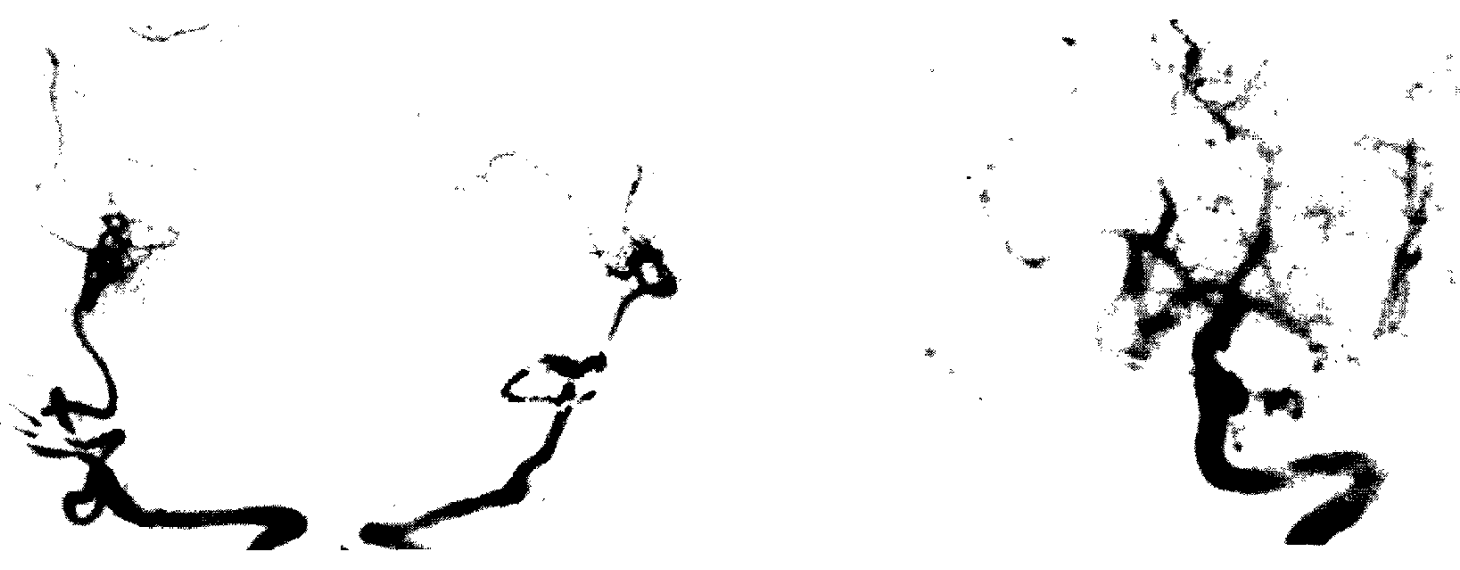

Fig. 4 Case 1. Postoperative angiograms revealing the complete clipping of both vertebral arteries. Good collateral circulation is present through the posterior communicating artery. left: Anteroposterior view of the left vertebral artery, center: anteroposterior view of the right vertebral artery, right: anteroposterior view of the left internal carotid artery. 
turbance gradually resolved. Four years later, she is doing well with right hearing disturbance but without other disorders. Postoperative angiography revealed complete occlusion of the aneurysm with collateral blood flow from the posterior communicating artery to the basilar artery without any contrast medium entering into the aneurysmal cavity (Fig. 4). Case 2: A 38-year-old male experienced left hemiparesis and dysarthria upon awakening. The symptoms disappeared completely within 24 hours. CT, MR imaging, and angiography at a local hospital disclosed a giant aneurysm at the basilar trunk. The symptoms were thought to be due to embolism from the aneurysm. The patient was treated at the hospital with anticoagulation therapy using ticlopidine (100 mg/day). Follow-up MR imaging and angiography one year later disclosed an increase in the aneurysm size and he was referred to our hospital (Figs. 5 and 6). Most of the nidus was filled with thrombus. The feasibility of neck clipping of this aneurysm was thought to be very low, because dissection of the perforating arteries from the aneurysm was considered very difficult. Prior to surgical intervention, test occlusion of the basilar artery was performed with hypotensive challenge (30\% decrease of systolic pressure with $\mathrm{Ca}^{2}$ antagonist). The balloon (superselective balloon catheter; Kaneka Medics, Osaka) was inflated at the basilar artery just proximal to the bilateral anterior inferior cerebellar arteries. The patient tolerated temporary occlusion of basilar artery for 15 minutes under normal pressure and also for 15 minutes under hypotension. Since surgery was thought to be

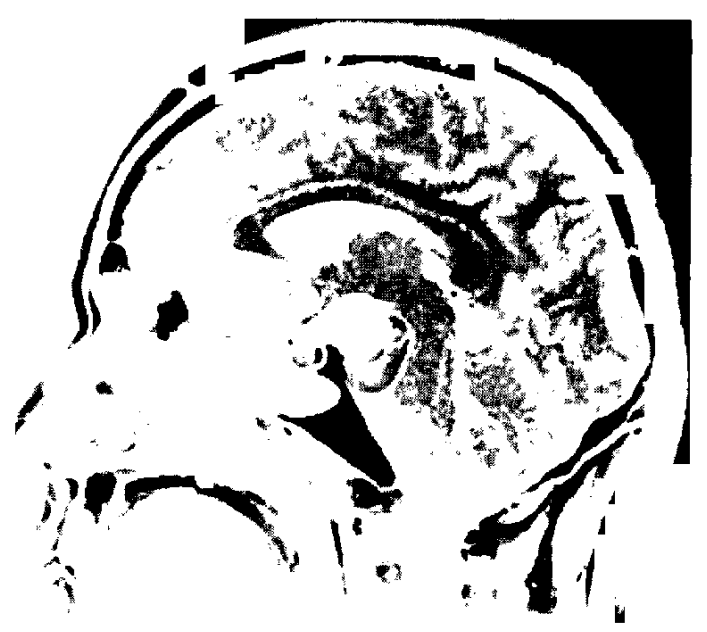

Fig. 5 Case 2. Sagittal magnetic resonance image showing a large and partially thrombosed aneurysm compressing the midbrain and pons.

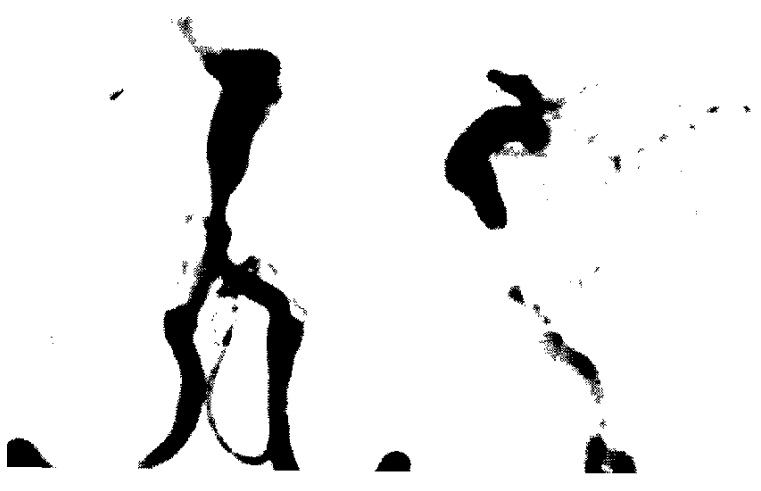

Fig. 6 Case 2. Left vertebral angiograms showing dilatation of the basilar artery. The aneurysm is distal to both anterior inferior cerebellar arteries. left: Anteroposterior view, right: lateral view.

very risky and the symptom was transient ischemic attack, the patient was discharged. He later experienced another transient ischemic attack while at work on a fishing boat. He expressed the desire to be treated and was readmitted one month later.

The patient was placed in the lateral position, and a right suboccipital craniotomy was performed. The aneurysm was very arteriosclerotic and the dissection was carried out carefully. The basilar artery, both anterior inferior cerebellar arteries, and the right side of the aneurysmal neck were exposed. The neck could not be clipped because of its width and the arteriosclerosis. Therefore, the basilar artery
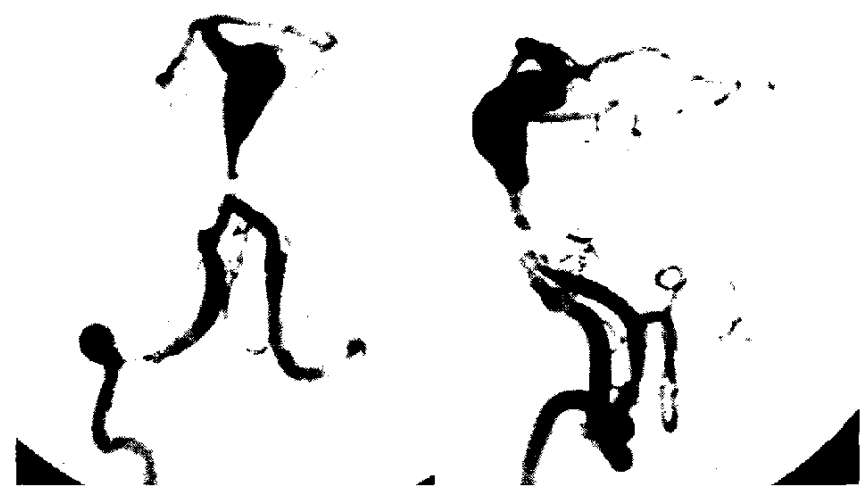

Fig. 7 Case 2. Postoperative vertebral angiograms showing the basilar artery remained open in spite of two clips applied just proximal to both anterior inferior cerebellar arteries. left: Anteroposterior view, right: lateral view. 
was clipped between the anterior inferior cerebellar artery and the aneurysmal neck. However, because the artery was very stiff and hard due to arteriosclerosis so the applied clip slipped proximally, obliterating both anterior inferior cerebellar arteries. Therefore, this clip was removed from this segment of the basilar artery and two clips were applied to the basilar artery proximal to the anterior inferior cerebellar artery where the arteriosclerosis appeared to be milder.

The postoperative course was uneventful except for slight right abducens, facial, and lower motor nerve pareses. Follow-up angiography disclosed that the clips had not completely occluded the arterial lumina (Fig. 7). Recraniotomy was considered but the arteriosclerosis of the basilar artery was so advanced that reclipping could not ensure occlusion of the segment. Thus endovascular treatment was planned. The basilar arterial segment distal from anterior inferior cerebellar artery appeared to be appropriate as the occlusion site, but this segment enlarges distally, so any occlusion material would migrate from this site to the distal aneurysm or the basilar artery. Also, the short segment of the basilar artery between the junction and the anterior inferior cerebellar artery appeared to be too short for occlusion, as the Dacron fiber coil was not commercially available at that time. In addition, this segment could not be visualized under the x-ray image intensifier because of the applied titanium clips, so controlled placement of the detachable coil was unlikely. Finally, occlusion of the aneurysmal cavity was planned.

A catheter was introduced into the vertebral artery under local anesthesia 26 days after the craniotomy. An electro-detachable balloon catheter was navigated through the arterial lumen. ${ }^{15}$ Four balloons were detached inside the aneurysm to completely obliterate the aneurysm (Fig. 8). Coils were prepared to assist the balloons in completely occluding the aneurysm, but were not required. After the procedure, heparin was continuously infused starting at $15,000 \mathrm{U} /$ day. The patient's condition was very good until the following afternoon when he developed right hemiparesis. CT showed no additional low-density or high-density area compared with the preoperative CT scan. Angiography revealed no change compared to the angiography performed immediately after endovascular treatment. Occlusion of the small perforating branches was thought to be the cause of the hemiparesis. Tissue-plasminogen activator $(5 \times$ $10^{6} \mathrm{U}$ ) was intraarterially injected into the basilar artery, and the hemiparesis disappeared immediately. Two hours later, his level of consciousness deteriorated and he became comatose. CT revealed a massive epidural hemorrhage at the region of the craniotomy performed (27 days before) (Fig. 9). An emergency operation was performed to remove the hematoma, but the patient's consciousness level did not recover well and he is now disabled.

Case 3: A 43-year-old female had sudden onset of headache. She was referred to a local hospital, where subarachnoid hemorrhage was found. CT and angiography revealed a giant aneurysm at the vertebral junction (Fig. 10).

The aneurysm was directed posteriorly and was considered difficult to clip. Intravascular treatment was attempted. The microcatheter was guided up to

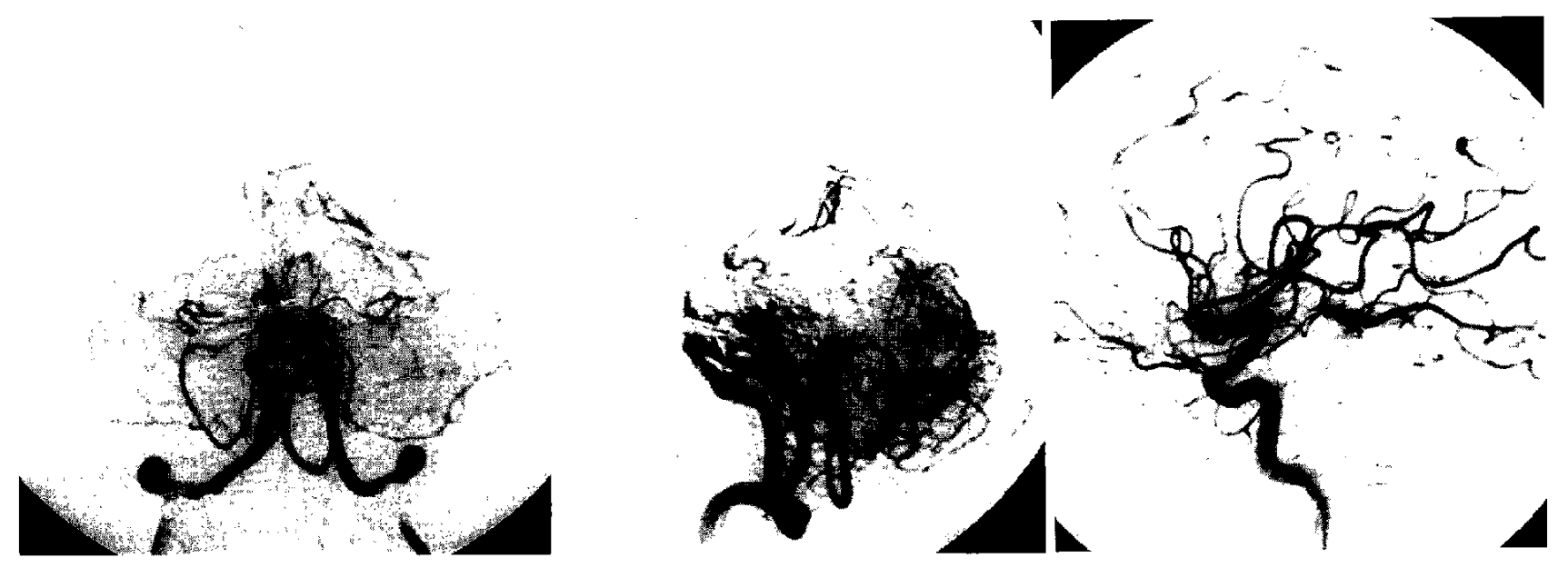

Fig. 8 Case 2. left, center: Vertebral angiograms after balloon occlusion of the aneurysm. left: Anteroposterior view, center: lateral view. right: Left internal carotid angiogram, lateral view. The left internal carotid artery provides collateral flow to the posterior cerebral artery. Complete occlusion of the aneurysm is observed. 


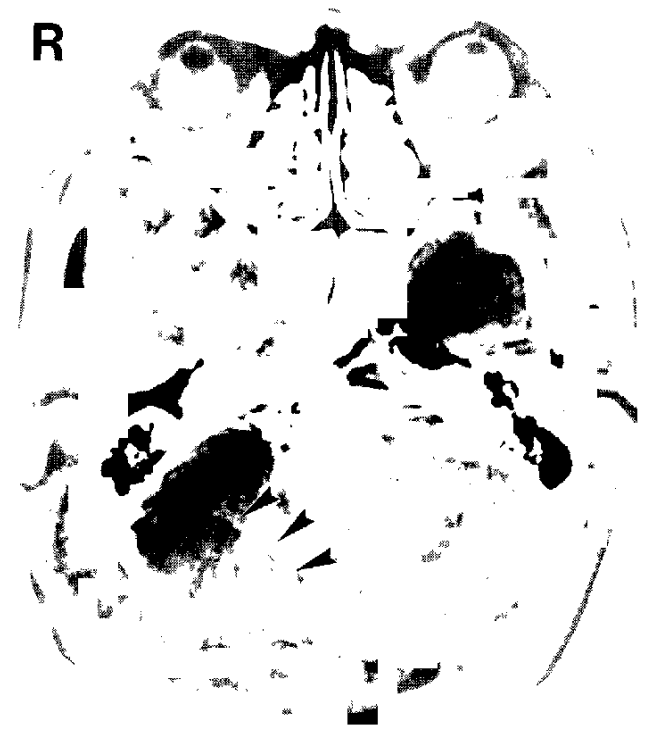

Fig. 9 Case 2. Computed tomography scan revealing a large epidural hematoma at the craniotomy site (arrowheads).

the aneurysmal cavity and the distal part of an interlocking detachable coil (Target Inc.) was introduced into the aneurysmal cavity, but the coil protruded into the basilar artery and had to be withdrawn. There was no possibility of occluding only the aneurysmal cavity and preserving the basilar arterial blood flow by an intravascular procedure. Angiography before the treatment showed that the bilateral posterior communicating arteries were not well opacified by the either right or left internal carotid angiography (Fig. 11 upper row). Temporary occlusion of the bilateral vertebral arteries caused nystagmus, dysarthria, and consciousness disturbance immediately after both vertebral arteries were occluded simultaneously. Auditory brain stem response showed prolongation of the latency of the Vth wave. Since the neurological deficit occurred immediately, internal carotid angiography was not performed during the temporary balloon occlusion to analyze the collateral blood flow through the posterior communicating arteries. If the aneurysm could not be clipped with preservation of the basilar arterial blood flow, brain stem infarction was anticipated due to obliteration of the basilar artery. Therefore, bypass was thought to be necessary.

A left suboccipital craniotomy combined with the presigmoid, transtentorial approach and far lateral approach was used to place a radial arterial graft between the vertebral artery and the posterior cerebral artery. During the craniotomy, the aneurysmal neck was examined carefully, but it was too large to be clipped, and finally the left vertebral artery was clipped. We did not clip both vertebral arteries at this stage to secure the patency of the bypass with sufficient blood flow through the graft. Moreover, intraoperative vertebral angiographic images in the lateral position cannot be obtained at our angiographic suite. Thus clipping of the right vertebral artery was postponed. Thirty-three days after the craniotomy, test occlusion of the right vertebral artery was performed with hypotensive challenge. The patient tolerated the test well in spite of the bypass closure. In contrast to the poor opacification of the posterior communicating arteries before left ver-
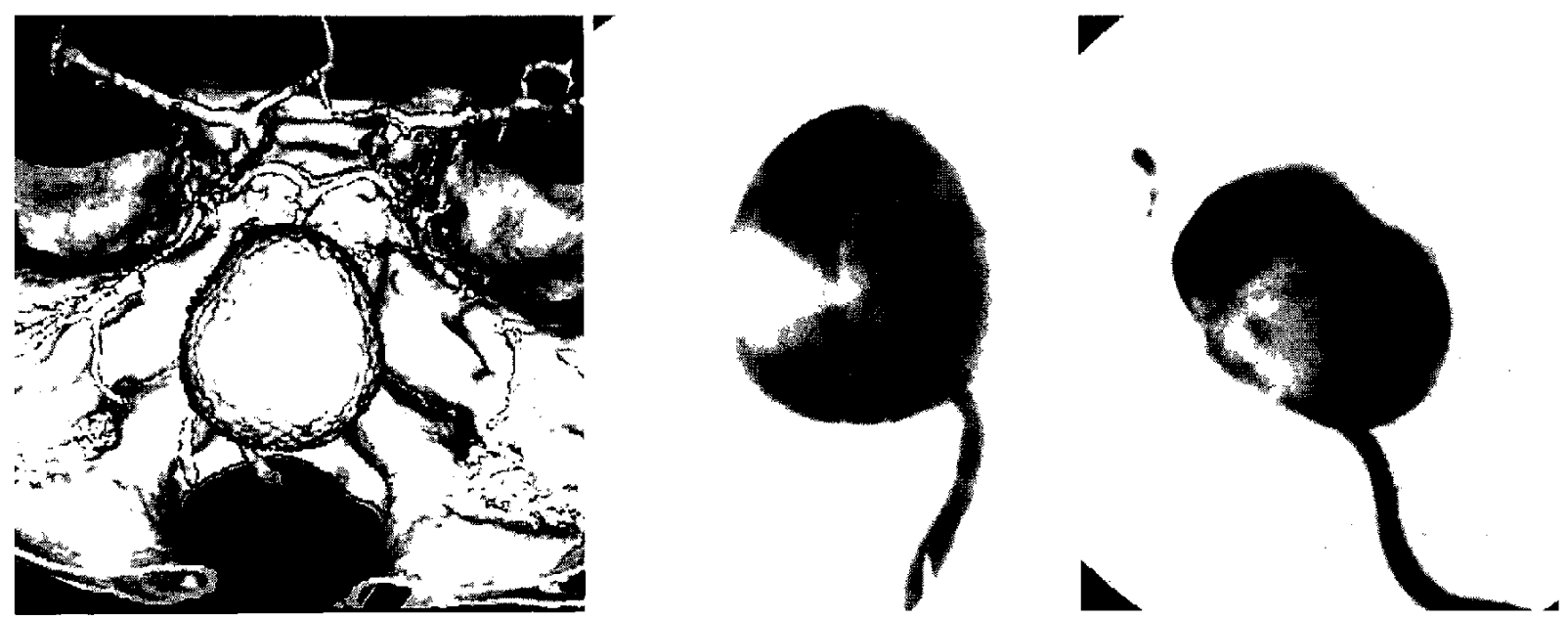

Fig. 10 Case 3. left: Three-dimensional computed tomography scan showing a giant aneurysm of the basilar artery. center, right: Left vertebral arteriograms showing a giant aneurysm measuring $4.5 \mathrm{~cm}$. center: Anteroposterior view, right: lateral view. 


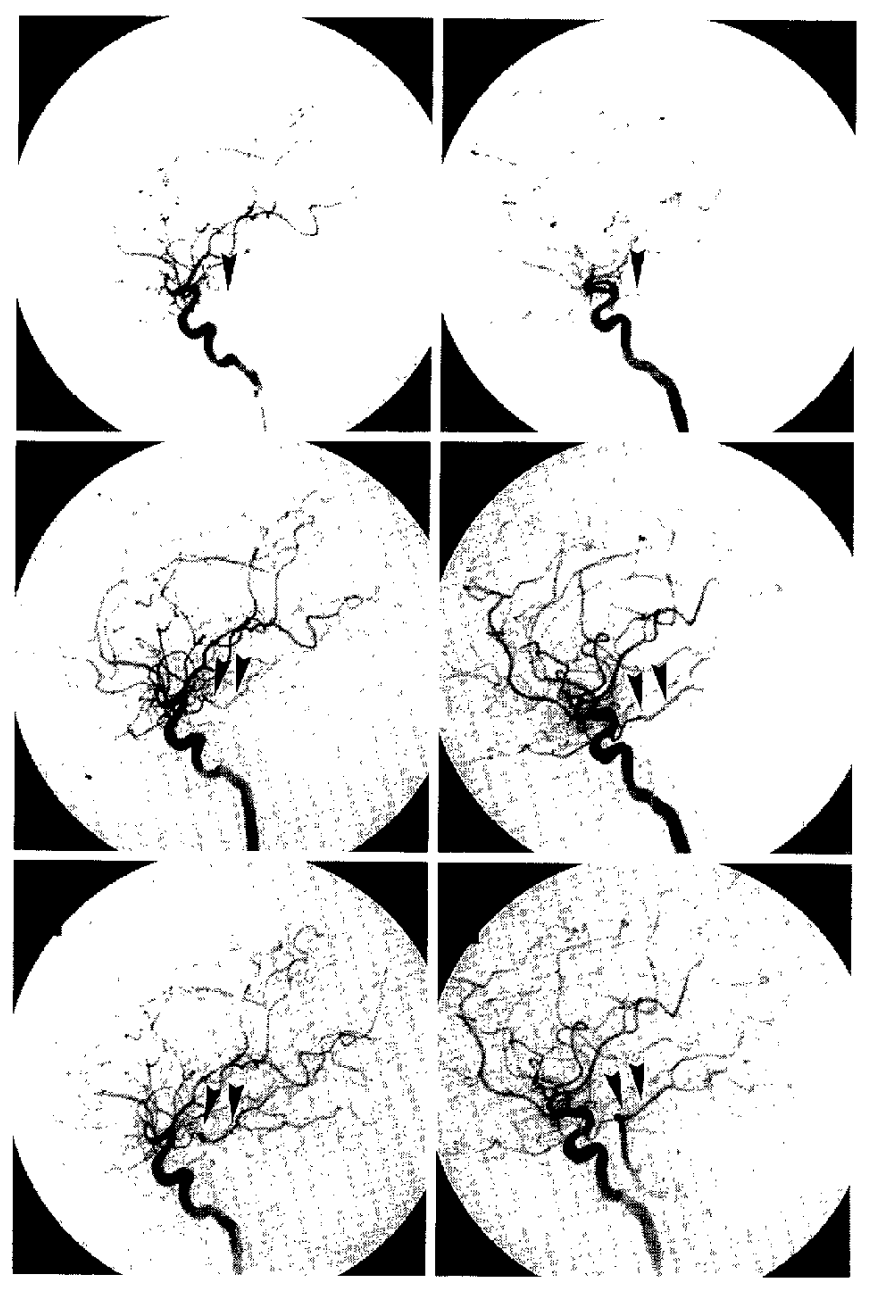

Fig. 11 Case 3. upper row: Right (left) and left (right) internal carotid angiograms before operation showing the small posterior communicating arteries (arrowhead). middle row: Right (left) and left (right) internal carotid angiograms 33 days after operation showing increased collateral blood flow through both posterior communicating arteries (arrowheads). lower row: Right (left) and left (right) internal carotid angiograms showing good collateral blood flow through the posterior communicating arteries (arrowheads) after temporary balloon occlusion of the right vertebral artery.

tebral artery occlusion, right and left internal carotid angiography showed enlarged posterior communicating arteries 33 days later, before the temporary balloon occlusion of the right vertebral artery (Fig. 11 middle row), and good collateral blood flow through both posterior communicating arteries after the temporary balloon occlusion of the right vertebral artery (Fig. 11 lower row). There was no prolongation of the latency of the Vth wave in the auditory brain stem response.

Fifty days after the craniotomy, the operative wound had healed well, so the right vertebral artery was obliterated with coils. Two detachable coils were placed in the right vertebral artery just proximal to the aneurysm. Two additional Dacron fiber coils were placed inside the two coils. The four coils completely obliterated the vertebral artery (Fig. 12 left, center). Thus patient had no posterior inferior cerebellar artery on either vertebral artery, so the main collateral flow to the basilar artery was from the bilateral internal carotid arteries through the posterior communicating arteries. Immediately after the intervention, angiography disclosed retrograde filling of the aneurysm as well as both anterior inferior cerebellar arteries.

Intravenous administration of heparin $(15,000$ U/day) was started and the dosage was gradually decreased within 7 days. Aspirin (81 mg/day) and ticlopidine $(100 \mathrm{mg} /$ day) were administered per os. Two days later, the patient developed right hemiparesis and right facial nerve paresis. Urokinase $(60,000 \mathrm{U})$ was administered intravenously, with complete disappearance of the symptoms, but 2 days later, the symptoms recurred and urokinase $(60,000$ U) was again administered intravenously, and again the symptoms disappeared. The cause of these transient ischemic attacks was thought to be either thrombosis at the orifice of the anterior inferior cerebellar artery or aneurysmal enlargement due to thrombosis inside the aneurysm. Hypoperfusion might have been involved but this was considered unlikely because hypotension was not observed during either transient ischemic attack. There was no neurological deficit thereafter. Serial CT studies disclosed progression of thrombosis of the aneurysm and when contrast enhancement of the aneurysm was no longer observed on day 7 , thrombosis of the aneurysm was thought to be complete. Follow-up angiography revealed no evidence of the aneurysm (Fig. 12 right). The patient was discharged without neurological deficit. Follow-up angiography one year later still showed complete obliteration of the aneurysm.

\section{Discussion}

Intravascular coil placement was attempted in Cases 1 and 3 but it protruded into the parent artery and had to be withdrawn. Coil placement was not attempted in Case 2 because the basilar arterial flow could not be spared by a detachable coil. Therefore, large and giant aneurysms at this location cannot be obliterated by interventional radiological proce- 

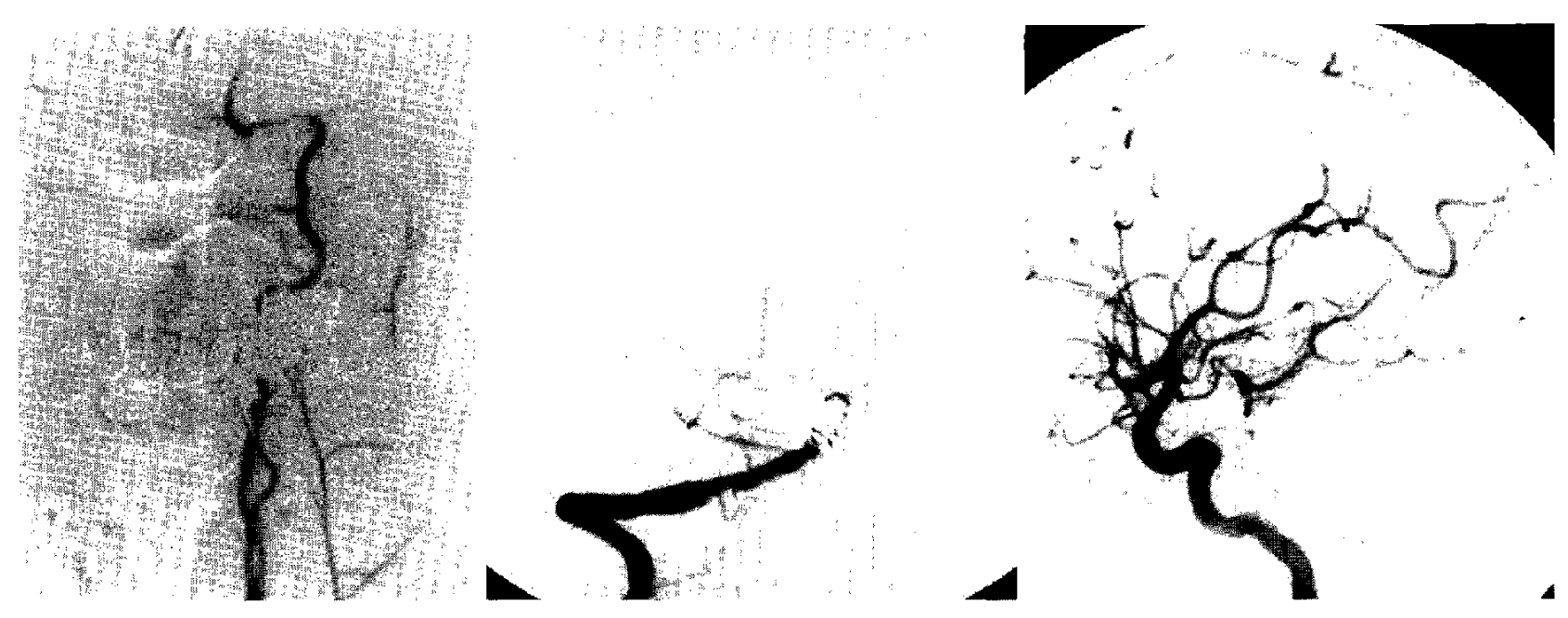

Fig. 12 Case 3 . left: Left vertebral angiogram revealing the occlusion of this artery by a surgical clip. No patency of the bypass graft is observed. center: Right vertebral angiogram showing occlusion with coils just proximal to the aneurysm. right: Right internal carotid angiogram showing no evidence of the aneurysm.

dures with preservation of the basilar arterial blood flow and parent artery occlusion thus must be considered. The balloon occlusion test must be performed, but the relationship between $\mathrm{rCBF}$ and vertebral arterial occlusion test is unknown. In our clinic, rCBF images are obtained with a single photon emission CT using HMPAO, but this do not provide quantitative data. A prominent decrease in rCBF was seen in Case 3, but the critical rCBF value for occlusion in the vertebrobasilar system is unknown because of technical difficulties in quantitative analysis of the posterior fossa CBF and the limited number of clinical cases. Thus $\mathrm{rCBF}$ images still provide only auxiliary data for evaluation of vertebral or basilar artery occlusion.

Temporary occlusion with depression of blood pressure appears to be a more practical test. Intravenous administration of $\mathrm{Ca}^{2+}$ antagonist or ganglion blocker can obtain a blood pressure as low as $70 \%$ of the normal systolic pressure. This perioperative test seems to enhance the safety of actual permanent arterial occlusion. In Cases 1 and 2, the blood pressure was lowered before permanent occlusion of the vertebral or basilar artery and the patients tolerated the hemodynamic reduction. However, Case 3 did not tolerate simultaneous occlusion of both vertebral arteries before treatment, so radial arterial graft was expected to provide better blood flow than superficial temporal artery-superior cerebellar artery or superficial temporal artery-posterior cerebral artery bypass surgery. However, bypass through the superficial temporal artery might have provided sufficient flow for the patient to tolerate the ischemic events after vertebral artery occlusion. One impediment to bypass in Case 3 was the positioning during surgery. The patient was placed lateral with her head rotated to the opposite side for a better view of the posterior fossa. This rather occipital approach in the lateral position makes the anterior subtemporal approach to the superior cerebellar artery trunk difficult because of the vein of Labbé which traverses the operative field. A more anterior subtemporal approach would have provided a better view for dissection of the main trunk of the superior cerebellar artery. Thus, use of the peripheral branch of the posterior cerebral artery that leads to the temporal base as a recipient was mandatory. This might have resulted in the occlusion of the bypass graft. Though the bypass was not patent, sufficient collateral blood flow to maintain the posterior fossa gradually developed within 33 days, which was very fortunate. One interesting observation in this case is that staged occlusion of the right and left vertebral arteries is sometimes made possible by the development of spontaneous collateral circulation in a patient who do not tolerate the occlusion test. ${ }^{13\}}$

Postoperative management must prevent thromboembolic complications in the tiny branches and perforators near or at the trunk. ${ }^{3)}$ We administered heparin at 10,000-15,000 U/day and maintained the activated clotting time at 1.5 fold that of the pretreatment time. Heparin administration was gradually decreased and withdrawn within 5 days. We also used aspirin to prevent thromboembolism. In spite of this treatment, two of our three patients developed postoperative ischemia which was thought to 
be due to thrombosis of perforators or small branches. Fibrinolytic agent was used with great efficacy to treat both patients. Unfortunately, one patient developed epidural hematoma at the craniotomy site. Another patient showed very prompt response to urokinase. Direct surgery carries a risk of hemorrhage at the craniotomy site as was seen in Case 2 because of possible repeated use of antifibrinolytic therapy. Intravascular treatment will allow more active anticoagulation therapy. Cranial nerve paresis is also common with surgical intervention. The major advantage of craniotomy is that clipping does not occlude a long segment of the parent artery and occlusion of perforators and branches can be avoided. Endovascular treatment cannot easily occlude a parent artery within a very short segment. However, carefully considered intravascular procedures may achieve occlusion within a short segment by using a cage of detachable coils and placing additional thrombogenic Dacron fiber coils inside the cage. Recent fine coils and intravascular techniques will allow occlusion of the parent artery at a finer scale and with greater confidence. This offers great advantages in preventing postocclusion thrombosis or embolism. Various anticoagulants can be used more safely after intravascular treatment than after craniotomy. Careful consideration and selection of various surgical and interventional techniques will allow a large or giant aneurysm located at the middle to lower basilar artery to be successfully treated.

\section{References}

1) Aymard A, Gobin P, Hodes JE, Bien $S$, Rufenacht $D$, Reizine D, George B, Merland JJ: Endovascular occlusion of vertebral arteries in the treatment of unclippable vertebrobasilar aneurysms. J Neurosurg 74: 393398, 1991

2) Baumgartner WA, Silverberg GD, Ream AK, Jamieson SW, Tarabek J, Reitz BA: Reappraisal of cardiopulmonary bypass with deep hypothermia and circulatory arrest for complex neurosurgical operations. Surgery 94: 242-249, 1983

3) Drake CG: Ligation of the vertebral (unilateral or bilateral) or basilar artery in the treatment of large intracranial aneurysms. J Neurosurg 43: 255-274, 1975

4) Drake CG, Barr HWK, Coles JC, Gergely NF: The use of extracorporeal circulation and profound hypothermia in the treatment of ruptured intracranial aneurysm. J Neurosurg 21: 575-581, 1964

5) Guglielmi G, Vinuela F, Dion J, Duckwiler G: Electrothrombosis of saccular aneurysm via endovascular approach II. Preliminary clinical experience. I Neurosurg 75: 8-14, 1991

6) Guglielmi G, Vinuela F, Sepetka I, Macellari V: Electrothrombosis of saccular aneurysm via endovascu- lar approach I. Electrochemical basis, technique, and experimental results. J Neurosurg 75: 1-7, 1991

7) Hayakawa $T$, Kamikawa K, Ohnishi $T$, Yoshimine $T$ : Prevention of postoperative complications after a transoral transclival approach to basilar aneurysms. $J$ Neurosurg 54: 699-703, 1981

8) Heros RC: Lateral suboccipital approach for vertebral and vertebrobasilar artery lesions. J Neurosurg 64: $559-562,1986$

9) Higashida RT, Halbach VV, Cahan LD, Hieshima GB, Konishi Y: Detachable balloon embolization therapy of posterior circulation intracranial aneurysms. $J$ Neurosurg 71: 512-519, 1989

10] Hopkins LN, Bundy JL, Castellani D: Extracranial-intracranial arterial bypass and basilar artery ligation in the treatment of giant basilar artery aneurysms. Neurosurgery 13: 189-194, 1983

11) Solomon RA, Smith CR, Raps EC, Young WL, Stone JG, Fink ME: Deep hypothermic circulatory arrest for the management of complex anterior and posterior circulation aneurysms. Neurosurgery 29: 732-738, 1991

12) Spetzler RF, Hadley MN, Rigamonti D, Carter LP, Raoudzens PA, Shedd SA, Wilkinson E: Aneurysms of the basilar artery treated with circulatory arrest, hypothermia, and barbiturate cerebral protection. $J$ Neurosurg 68: 868-879, 1988

13) Steinberg GK, Drake CG, Peerless SI: Deliberate basilar or vertebral artery occlusion in the treatment of intracranial aneurysms. J Neurosurg 79: 161-173, 1993

14) Sugita K, Kobayashi S, Shintani A, Mutsuga N: Microneurosurgery for aneurysms of the basilar artery. J Neurosurg 51: 615-620, 1979

15) Taki W, Handa $H$, Yamagata $S$, Yonekawa $Y$, Iwata $H$, Ikada $Y$ : The releasable balloon technique with activated high frequency electrical current. Surg Neurol 13: 405-408, 1980

16) Yamaura A, Makino $H$, Isobe $K$, Nakamura $T$, Takemiya S: Repair of cerebrospinal fluid fistula following transoral transclival approach to a basilar aneurysm. Technical note. J Neurosurg 50: 834-836, 1979

17) Yasargil MG, Antic J, Laciga R, Jain KK, Hodosh RM, Smith RD: Microsurgical pterional approach to aneurysms of the basilar bifurcation. Surg Neurol 6: 83-91, 1976

Address reprint requests to: $W$. Taki, M.D., Department of Neurosurgery, Kyoto University Medical School, 54 Kawahara-cho, Shogoin, Sakyo-ku, Kyoto 606-8507, Japan.

\section{Commentary}

This series of three patients with large or giant aneurysms of the vertebral confluence or proximal basilar trunk illustrates the difficulties in treating these challenging lesions. Unfortunately, endovascular technology has not yet provided an ideal option for 
obliterating these large sacs and preserving the parent vessel.

For optimal treatment of these aneurysms, individual aneurysm angioarchitecture, degree of collateral blood flow, and patient neurological status must all be taken into consideration in creating an individualized therapeutic plan for each patient. As suggested by this series, a multi-disciplinary approach, creativity and willingness to alter the therapeutic plan are necessary elements for a successful outcome.

Ultimately, I believe the development of small, flexible stents will serve as another tool in the armamentarium for the neurovascular team treating these complex lesions.

Daniel L. BARROW, M.D. Department of Neurosurgery The Emory Clinic, Inc. Georgia, U.S.A.

Dr. Taki and his colleagues are to be commended for describing multimodality treatment results of large and giant aneurysm of the middle or lower basilar trunk. Despite the evolution of skull base surgical techniques, intra-aneurysmal treatment, and hypothermic circulatory arrest, large and giant basilar trunk aneurysms remain as one of the most difficult lesions for cerebrovascular surgeons. Consequently, most of these lesions had to be treated by combined surgical and interventional techniques sacrificing the parent artery with considerable morbidity. However, counting on rapid development of interventional technology, the most promising answer (obliteration of aneurysm with preservation of parent arteries and perforators) for these wide-necked, arteriosclerotic, or thrombotic large and giant basilar trunk aneurysms would be intra-aneurysmal treatment with detachable coils after remodeling of the wide aneurysm neck, or use of a tailored stent.

\section{Kyu Chang LEE, M.D. \\ Department of Neurosurgery Yonsei University College of Medicine Seoul, Korea, R.O.K.}

The authors reported 3 cases with vertebrobasilar giant aneurysm. The first case with an aneurysm of the vertebral artery junction tolerated the balloon occlusion test well and was finally treated by proximal occlusion of the parent arteries. The second case had a basilar trunk aneurysm and became disabled because of intracerebral hemorrhage after endovascular treatment. The third patient did not tolerate the occlusion test at first, but became tolerant after bypass surgery with a radial artery graft. The second case implies difficulty in postoperative management after endovascular surgery. Treatment for such lesions is very difficult and always challenging. Neurosurgeons should consider any possible methods. Combination of direct surgery, endovascular surgery, and bypass surgery is essential. Staged approach may be useful, because remodeling of the vascular supply may be expected after bypass surgery as shown in the third case. This paper is encouraging.

Hideaki NuKUI, M.D. Department of Neurosurgery Yamanashi Medical University Yamanashi, Japan

A large or giant aneurysm in the posterior circulation is still a big challenge for neurosurgeons because of the technical difficulty involved in clipping the aneurysmal neck or possible ischemic complications after using the proximal occlusion method. Although endovascular intraaneurysmal embolization with detachable coils is one of the therapeutic options, potential problems still remain such as incomplete intraaneurysmal occlusion, or recanalization which is not infrequently seen even after the embolization was thought to have been complete. Also, it is not known for sure whether the mass sign of the giant aneurysm will resolve or not by this procedure.

The authors treated three cases with angiographically evaluated and acceptable clinical results. Their opinion that proximal occlusion should be considered for such lesions is quite acceptable, and the three reported cases were not considered to be candidates for intraaneurysmal coil embolization because of the possibility of recanalization and possible aneurysmal growth after the embolization. This article describes well the difficulty involved in anticipating an ischemic complication after proximal occlusion, even with use of CBF study. In Case 1, in spite of good tolerance during the bilateral vertebral artery occlusion test, ischemic complication occurred after bilateral vertebral artery occlusion. In Case 3, however, a good collateral blood flow was present via the bilatera] posterior communicating arteries to the lower basilar artery, in spite of intolerance to test occlusion of the bilateral vertebral arteries.

Difficulty in the treatment of large or giant aneurysms in the posterior circulation is also suggested even with combined treatment using endovascular and direct surgical techniques.

Shigeharu SuzukI, M.D. Department of Neurosurgery Hirosaki University School of Medicine Aomori, Japan 